\title{
INTEGRATION OF TRIVARIATE POLYNOMIALS OVER LINEAR POLYHEDRA IN EUCLIDEAN THREE-DIMENSIONAL SPACE
}

\author{
H. T. RATHOD ${ }^{1}$ and H. S. GOVINDA RAO
}

(Received 13 April 1995; revised 24 October 1995)

\begin{abstract}
This paper concerns with analytical integration of trivariate polynomials over linear polyhedra in Euclidean three-dimensional space. The volume integration of trivariate polynomials over linear polyhedra is computed as sum of surface integrals in $\mathbb{R}^{3}$ on application of the well known Gauss's divergence theorem and by using triangulation of the linear polyhedral boundary. The surface integrals in $\mathbb{R}^{3}$ over an arbitrary triangle are connected to surface integrals of bivariate polynomials in $\mathbb{R}^{2}$. The surface integrals in $\mathbb{R}^{2}$ over a simple polygon or over an arbitrary triangle are computed by two different approaches. The first algorithm is obtained by transforming the surface integrals in $\mathbb{R}^{2}$ into a sum of line integrals in a one-parameter space, while the second algorithm is obtained by transforming the surface integrals in $\mathbb{R}^{2}$ over an arbitrary triangle into a parametric double integral over a unit triangle. It is shown that the volume integration of trivariate polynomials over linear polyhedra can be obtained as a sum of surface integrals of bivariate polynomials in $\mathbb{R}^{2}$. The computation of surface integrals is proposed in the beginning of this paper and these are contained in Lemmas 1-6. These algorithms (Lemmas 1-6) and the theorem on volume integration are then followed by an example for which the detailed computational scheme has been explained. The symbolic integration formulas presented in this paper may lead to an easy and systematic incorporation of global properties of solid objects, for example, the volume, centre of mass, moments of inertia etc., required in engineering design processes.
\end{abstract}

\section{Introduction}

The computation of area, volume, centre of mass, moment of inertia and other geometrical properties of rigid homogeneous solids are of central interest in a large number of engineering applications, in CAD/CAE/CAM applications, in geometric modelling and as well as in robotics. Computation of such physical quantities is defined by triple integrals over domains of three-dimensional Euclidean space. Quadrature formulas for multiple integrals have always been of great interest in computer applications.

\footnotetext{
'Department of Mathematics, Central College Campus, Bangalore University, Bangalore-1, India (C) Australian Mathematical Society, 1998, Serial-fee code 0334-2700/98
} 
A good overview of various methods for evaluating volume (triple) integrals in this context is given by Lee and Requicha [4]. Lee and Requicha observe that most computational studies in multiple integration often deal with calculations over very simple domains, such as a cube or a sphere, while the integrating function is very complicated, on the contrary in most of the engineering applications the converse problem usually arises. In such problems the integration domain may have a nonconvex shape and the function inside the integral sign is a trivariate polynomial. Lee and Requicha [5] outlined a family of approximate algorithms for computing inertial properties of solids. Such algorithms are based on a representation conversion from CSG to octree, via recursive subdivision. With a different approach based on the concept of finite elements, O'Leary [7] developed integration formulas based on a quasi disjoint decomposition of the solid in volume elements of simple predefined shape. Wilson and Farrior [9] gave a large set of formulas for the computation of the main geometrical and inertial properties of planar polygons and of rotational solids. Timmer and Stern [8] discussed a theoretical approach to the evaluation of volume integrals by transforming the volume integral to a surface integral over the boundary of the integration domain. Lien and Kajiya [6] presented an outline of a closed formula of volume integration for a tetrahedron and suggested that volume integration for a linear polyhedron can be obtained by decomposing it into a set of solid tetrahedrons. Cattani and Paoluzzi [2,3] gave a symbolic solution to both surface and volume integration of trivariate polynomials in $\mathbb{R}^{3}$ by using a triangulation of the solid based on the concept proposed by Timmer and Stern [8]. In a recent paper, Bernardini [1] presented the evaluation of integrals over $n$-dimensional linear polyhedra which are based on methods proposed earlier by Timmer and Stern [8] and Lien and Kajiya [6].

In the present paper, we have developed closed-form integration formulas which mainly follow the concepts proposed by Timmer and Stern [8] and Cattani and Paoluzzi [2, 3]. In the derivation of these formulas, our approach differs from that of Cattani and Paoluzzi in that we have transformed the surface integral in three-space to a double integral over a polygon in the $x y$-plane via the use of the equation of a plane spanning the three co-ordinates of a triangle in three-space. The double integral in plane over a polygon can then be computed by either transforming it into unit triangles in two-parameter spaces or by means of line integrals along the edges of the two-dimensional polygons. We have also addressed these problems, with an aim to give more efficient algorithms than previous work of Cattani and Paoluzzi [2, 3]. In these derivations, we have made reference to the well known Gauss's Divergence theorem, Taylor series expansion and Leibnitz theorem on differentiation to produce the present form of analytical integration formulas. It is very clear from these formulas that they do not duplicate the integration over a unit triangle or over the edges of an arbitrary triangle which is in contrast to the previous studies $[2,3]$ and thus save in terms of arithmetic operations. The theorem connecting a volume integral to 
a surface integral in three-space and in turn further connecting a surface integral in three-space to a double integral in two-space is of prime importance and leads to the closed-form integration formulas presented in Section 3 of this paper. Two different algorithms proposed in this paper to compute the volume integral in three-space is evident as a natural consequence of the Lemmas 1-5 and Lemma 6 on computing the surface (double) integrals in two-space which are discussed in Section 2. These explicit integration formulas are followed by an application example in Section 4 of this paper for which we have explained the detailed computational scheme using the two algorithms proposed in Sections 2 and 3.

\section{Surface integration}

In this section, we first establish two preliminary results which give closed-form analytical formulas for surface integration over a plane polygon in the $x y$-plane. Our aim is then to derive a closed formula for surface integration over a linear polyhedral surface in $\mathbb{R}^{3}$.

2.1. Let $\pi$ be a simple polygon in the $x y$-plane. We are actually interested in evaluating the integrals of the following kind, which we call the structure product over a linear two-polyhedron, that is, over the simple polygon in the $x y$-plane (see Fig. 1).

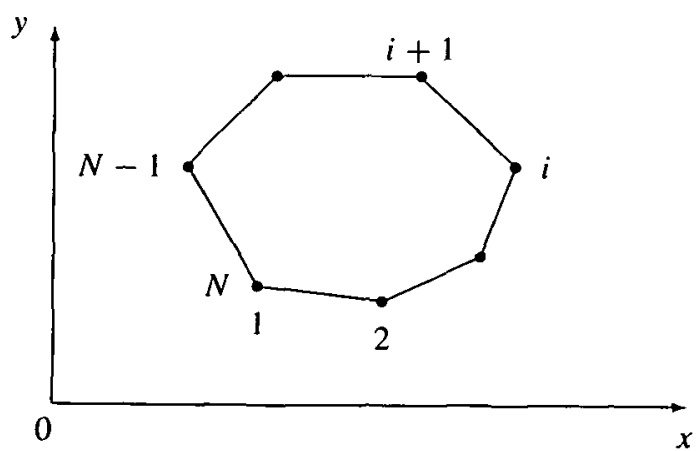

FIGURE 1. A simple polygon in the $x y$-plane.

We define

$$
I I_{\pi}^{\alpha . \beta . \gamma}(l, m, h)=\iint_{\pi} x^{\alpha} y^{\beta}(l x+m y+h)^{\gamma+1} d x d y
$$

where, $l, m, h$, are arbitrary real constants and $\alpha, \beta, \gamma$ are either zero or positive integers. 
LEMMA 1. The structure product $I I_{\pi}^{\alpha, \beta, \gamma}(0, m, h)$ over a simple polygon $\pi$ with $N$ oriented edges $l_{i, i+1}(i=1,2, \ldots, N)$ each with end points $\left(x_{i}, y_{i}\right)$ and $\left(x_{i+1}, y_{i+1}\right)$ in the $x y$-plane is expressible as

$$
I I_{\pi}^{\alpha, \beta . \gamma}(0, m, h)=\iint_{\pi} x^{\alpha} y^{\beta}(m y+h)^{\gamma+1} d x d y=\sum_{i=1}^{N} I_{L}^{i, i+1},
$$

where

(i)

$$
I_{L}^{i . i+1}=0 ; \quad \text { when } y_{i}=y_{i+1},
$$

(ii)

$$
I_{L}^{i, i+1}=\frac{y_{i, i+1}}{(\alpha+1)} \sum_{j=0}^{\alpha+\beta+\gamma+2} \frac{\lambda_{i, i+1}(j)}{j+1} \text { when } y_{i} \neq y_{i+1}, x_{i} \neq x_{i+1}
$$

and

$$
\begin{array}{r}
\lambda_{i, i+1}(j)=\sum_{j_{1}=0}^{j} \sum_{j_{2}=0}^{j-j_{1}} F_{i, i+1}\left(\alpha+1-j_{1}, j_{1}\right) G_{i, i+1}\left(\beta-j_{2}, j_{2}\right) \\
\times H\left(\gamma+1-j+j_{1}+j_{2}, j-j_{1}-j_{2}\right),
\end{array}
$$

(iii)

$$
I_{L}^{i, i+1}=y_{i, i+1} \frac{x_{i}^{\alpha+1}}{(\alpha+1)} \sum_{j=0}^{\beta+\gamma+1} \frac{\lambda_{i, i+1}(j)}{j+1} \quad \text { when } y_{i} \neq y_{i+1}, x_{i} \neq x_{i+1}
$$

and

$$
\lambda_{i, i+1}(j)=\sum_{j_{1}=0}^{j} G_{i, i+1}\left(\beta-j_{1}, j_{1}\right) H_{i, i+1}\left(\gamma+1-j+j_{1}, j-j_{1}\right)
$$

with

$$
\begin{aligned}
& F_{i, i+1}(r, s)=\frac{x_{i}^{r} x_{i, i+1}^{s}}{\underbrace{r} L^{s}} \stackrel{r s}{L}=\left(\begin{array}{c}
r+s \\
r
\end{array}\right) x_{i}^{r} x_{i, i+1}^{s}, \\
& G_{i, i+1}(r, s)=\frac{y_{i}^{r} y_{i, i+1}^{s}}{\underbrace{r}\lfloor s}\left\lfloor r s=\left(\begin{array}{c}
r+s \\
r
\end{array}\right) y_{i}^{r} y_{i, i+1}^{s}\right. \text {, } \\
& H_{i, i+1}(r, s)=\frac{z_{i}^{r} z_{i, i+1}^{s}}{\underbrace{r}\lfloor s}\left\lfloor r s=\left(\begin{array}{c}
r+s \\
r
\end{array}\right) z_{i}^{r} z_{i, i+1}^{s},\right. \\
& x_{i, i+1}=x_{i+1}-x_{i}, \quad y_{i, i+1}=y_{i+1}-y_{i}, \quad x_{i, i+1}=z_{i+1}-z_{i} .
\end{aligned}
$$


PROOF. We can write

$$
\begin{aligned}
I I_{\pi}^{\alpha, \beta . \gamma}(0, m, h) & =\iint_{\pi} x^{\alpha} y^{\beta}(m y+h)^{\gamma+1} d x d y \\
& =\iint_{\pi} \frac{\partial}{\partial x}\left\{\frac{x^{\alpha+1} y^{\beta}(m y+h)^{\gamma+1}}{(\alpha+1)}\right\} d x d y \\
& =\iint_{\pi}(\nabla \cdot \mathbf{F}) d x d y \\
\text { where } \quad \mathbf{F} & =\left(\frac{x^{\alpha+1} y^{\beta}(m y+h)^{\gamma+1}}{\alpha+1}, 0\right) .
\end{aligned}
$$

By applying the divergence theorem in two dimensions it follows that

$$
I I_{\pi}^{\alpha, \beta, \gamma}(0, m, h)=\iint_{\pi} x^{\alpha} y^{\beta}(m y+h)^{\gamma+1} d x d y=\oint_{\partial \pi} \frac{x^{\alpha+1} y^{\beta}(m y+h)^{\gamma+1}}{(\alpha+1)} d y .
$$

Let the boundary of the polygon $\partial \pi$ consist of $N$-line segments $l_{i, i+1}$ $(i=1,2, \ldots, N)$ each with end points at $\left(x_{i}, y_{i}\right)$ and $\left(x_{i+1}, y_{i+1}\right)$. It is clear that $\left(x_{1}, y_{1}\right)=\left(x_{N}, y_{N}\right)$ for the simple polygon of Figure 1 the parametric equations of the line segments $l_{i, i+1}$ may be taken as

$$
\begin{array}{ll}
x=x(t)=x_{i}+x_{i, i+1} t, & y=y(t)=y_{i}+y_{i, i+1} t, \\
z=z(t)=z_{i}+z_{i, i+1} t, & 0 \leq t \leq 1,
\end{array}
$$

where

$$
x_{i, i+1}=x_{i+1}-x_{i}, \quad y_{i, i+1}=y_{i+1}-y_{i}, \quad z_{i}=x_{m} y_{i}+h, \quad z_{i, i+1}=z_{i+1}-z_{i},
$$

Using (11), (12) and (13), we can write

$$
I I_{\pi}^{\alpha, \beta . \gamma}(0, m, h)=\sum_{i=1}^{N} I_{L}^{i . i+1},
$$

where $\quad I_{L}^{i, i+1}=\oint_{l_{i, i+1}} \frac{u(t) v(t) w(t)}{\alpha+1} d y(t)$

$$
=\frac{y_{i, i+1}}{(\alpha+1)} \int_{0}^{1} u(t) v(t) w(t) d t
$$

and $\quad u(t)=x^{\alpha+1}(t), \quad v(t)=y^{\beta}(t), \quad w(t)=z^{\gamma+1}(t)$. 
Using a Taylor series expansion of a function of a single variable $t$ about $t=0$ we can write

$$
f(t)=\sum_{j=0}^{\alpha+\beta+\gamma+2} \frac{f^{(j)}(0)}{\lfloor j} t^{j}=u(t) v(t) w(t),
$$

where

$$
f^{(j)}(0)=\left\{\frac{d^{j} f(t)}{d t^{j}}\right\}_{t=0} .
$$

Using Leibnitz's theorem on differentiation, it can be shown that

$$
\frac{f^{j}(0)}{\lfloor j}=\sum_{j_{1}=0}^{j} \sum_{j_{2}=0}^{j-j_{1}} \frac{u^{\left(j_{1}\right)}(0) v^{\left(j_{2}\right)}(0) w^{\left(j-j_{1}-j_{2}\right)}(0)}{\left\lfloorj _ { 1 } j _ { 2 } \left\lfloor j-j_{1}-j_{2}\right.\right.}
$$

and from (17) and (12), it can be further shown that

$$
\begin{aligned}
& \frac{u^{\left(j_{1}\right)}(0)}{\left\lfloor j_{1}\right.}=F_{i, i+1}\left(\alpha+1-j_{1}, j_{1}\right), \quad \frac{v^{\left(j_{2}\right)}(0)}{\left\lfloor j_{2}\right.}=G_{i, i+1}\left(\beta-j_{2}, j_{2}\right), \\
& \frac{w^{\left(j-j_{1}-j_{2}\right)}(0)}{j-j_{1}-j_{2}}=H_{i, i+1}\left(\gamma+1-j+j_{1}+j_{2}, j-j_{1}-j_{2}\right),
\end{aligned}
$$

where

$$
\begin{aligned}
F_{i, i+1}\left(\alpha+1-j_{1}, j_{1}\right) & =\frac{x_{i}^{\alpha+1-j_{1}} x_{i, i+1}^{j_{1}}}{\alpha+1-j_{1} \mid j_{1}}\lfloor\alpha+1 \\
G_{i, i+1}\left(\beta-j_{2}, j_{2}\right) & =\frac{y_{i}^{\beta-j_{2}} y_{i, i+1}^{j_{2}}}{\beta-j_{2}\left\lfloor j_{2}\right.} \mid \beta \\
H_{i, i+1}\left(\gamma+1-j+j_{1}+j_{2}, j-j_{1}-j_{2}\right) & =\frac{z_{i}^{\gamma+1-j-j_{1}-j_{2}} z_{i, i+1}^{j-j_{1}-j_{2}}}{\mid \gamma+1-j+j_{1}+j_{2}\left\lfloor j-j_{1}-j_{2}\right.}(22)
\end{aligned}
$$

Substituting from (21) into (20), we find

$$
\frac{f^{(j)}(0)}{\lfloor j}=\lambda_{i, i+1}(j)
$$

where

$$
\begin{aligned}
\lambda_{i, i+1}(j)=\sum_{j_{1}=0}^{j} \sum_{j_{2}=0}^{j-j_{1}} & F_{i, i+1}\left(\alpha+1-j_{1}, j_{1}\right) G_{i, i+1}\left(\beta-j_{2}, j_{2}\right) \\
& \times H_{i, i+1}\left(\gamma+1-j+j_{1}+j_{2}, j-j_{1}-j_{2}\right) .
\end{aligned}
$$


Integrating (18) with respect to $t$ from 0 to 1 , we get

$$
\begin{aligned}
\int_{0}^{1} u(t) v(t) w(t) d t & =\sum_{j=0}^{\alpha+\beta+\gamma+2} \frac{f^{(j)}(0)}{\lfloor j} \frac{1}{(j+1)} \\
& =\sum_{j=0}^{\alpha+\beta+\gamma+2} \frac{\lambda_{i, i+1}(j)}{(j+1)}
\end{aligned}
$$

Finally from (16) and (26), we see that

$$
l_{L}^{i, i+1}=\frac{y_{i, i+1}}{(\alpha+1)} \sum_{j=0}^{\alpha+\beta+\gamma+2} \frac{\lambda_{i, i+1}(j)}{j+1} .
$$

Clearly (27) is valid for all values of $x_{i}, x_{i+1}, y_{i}$ and $y_{i+1}$, but it assumes a simple value for $y_{i}=y_{i+1}$, viz.,

$$
I_{L}^{i, i+1}=0, \quad \text { when } y_{i}=y_{i+1} .
$$

This proves the results of (3) and (4). Proceeding in a similar manner, the results (6) and (7) can be easily proved.

The proofs of Lemmas (2-5) are similar to that of Lemma 1 and hence we include only the statements of the Lemmas.

LEMMA 2. The structure product $I I_{\pi}^{\alpha, \beta, \gamma}(l, 0, h)$ over a simple polygon $\pi$ with $N$ oriented edges $l_{i, i+1}(i=1,2, \ldots, N)$ each with end points $\left(x_{i}, y_{i}\right)$ and $\left(x_{i+1}, y_{i+1}\right)$ in the $x y$-plane is expressible as

$$
I I_{\pi}^{\alpha, \beta, \gamma}(l, 0, h)=\iint_{\pi} x^{\alpha} y^{\beta}(l x+h)^{\gamma+1} d x d y=-\sum_{i=1}^{N} I_{L}^{i, i+1},
$$

where

(i)

$$
I_{L}^{i, i+1}=0, \quad \text { when } x_{i}=x_{i+1}
$$

(ii)

$$
I_{L}^{i, i+1}=\frac{x_{i, i+1}}{(\beta+1)} \sum_{j=0}^{\alpha+\beta+\gamma+2} \mu_{i, i+1}(j) /(j+1) \quad \text { when } x_{i} \neq x_{i+1}, y_{i} \neq y_{i+1},
$$

and

$$
\begin{array}{rl}
\mu_{i, i+1}(j)=\sum_{j_{1}=0}^{j} \sum_{j_{2}=0}^{j-j_{1}} & F\left(\alpha-j_{1}, j_{1}\right) G\left(\beta+1-j_{2}, j_{2}\right) \\
& \times H\left(\gamma+1-j+j_{1}+j_{2}, j-j_{1}-j_{2}\right),
\end{array}
$$


(iii)

$$
I_{L}^{i, i+1}=x_{i, i+1} \frac{y_{i}^{\beta+1}}{(\beta+1)} \sum_{j=0}^{\alpha+\gamma+1} \mu_{i, i+1}(j) /(j+1) \quad \text { when } y_{i}=y_{i+1}, x_{i} \neq x_{i+1}
$$

and

$$
\mu_{i, i+1}(j)=\sum_{j_{1}=0}^{j} F_{i, i+1}\left(\alpha-j_{1}, j_{1}\right) H\left(\gamma+1-j+j_{1}, j-j_{1}\right),
$$

with

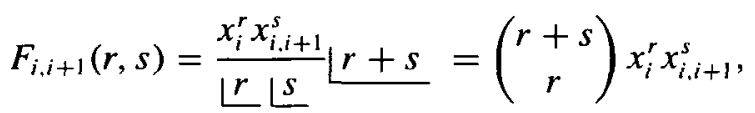

$$
\begin{aligned}
& G_{i, i+1}(r, s)=\frac{y_{i}^{r} y_{i, i+1}^{s}}{r} \underline{r} L r+s=\left(\begin{array}{c}
r+s \\
r
\end{array}\right) y_{i}^{r} y_{i, i+1}^{s} \text {, } \\
& H_{i, i+1}(r, s)=\frac{z_{i}^{r} z_{i, i+1}^{s}}{\underline{\underline{r}\lfloor s}\lfloor r+s}=\left(\begin{array}{c}
r+s \\
r
\end{array}\right) z_{i}^{r} z_{i, i+1}^{s}, \\
& x_{i, i+1}=x_{i+1}-x_{i}, \quad y_{i, i+1}=y_{i+1}-y_{i}, \quad z_{i}=l x_{i}+h, \quad z_{i, i+1}=z_{i+1}-z_{i} .
\end{aligned}
$$

LEMMA 3. The structure product $I I_{\pi}^{\alpha . \beta . \gamma}(l, m, 0)$ (where $l \neq 0, m \neq 0$ are arbitrary real constants and $\alpha, \beta, \gamma+1$ are either zero or positive integers) over a simple polygon with $N$-oriented edges $l_{i, i+1}(i=1,2, \ldots, N)$ each with end points at $\left(x_{i}, y_{i}\right)$ and $\left(x_{i+1}, y_{i+1}\right)$ in the $x y$-plane, is expressible as

$$
I I_{\pi}^{\alpha, \beta, \gamma}(l, m, 0)=\iint_{\pi} x^{\alpha} y^{\beta}(l x+m y)^{\gamma+1} d x d y=\sum_{i=1}^{N} I_{L}^{i, i+1},
$$

where

(i)

$$
I_{L}^{i . i+1}=0, \quad \text { when } y_{i}=y_{i+1}
$$

(ii)

$$
\begin{gathered}
I_{L}^{i, i+1}=y_{i, i+1} \sum_{j=0}^{\gamma+1} \Phi_{j, \gamma+1-j}(l, m) x_{i}^{\alpha+j+1} F^{i, i+1}(0, \beta+\alpha+1-j), \\
\text { when } y_{i} \neq y_{i+1}, \quad x_{i}=x_{i+1},
\end{gathered}
$$


(iii)

$$
\begin{aligned}
I_{L}^{i, i+1}= & y_{i, i+1} \Phi_{0, \gamma+1}(l, m) F(\alpha+1, \beta+\gamma+1) \\
& +Z(\gamma) \sum_{p=1}^{(\gamma+1) / 2} y_{i, i+1} A_{i}(2 p-1,2 p) F^{i, i+1}(\alpha+2 p, \beta+\gamma+1-2 p) \\
& \quad+B_{i, i+1}(2 p-1,2 p) H^{i, i+1}(\alpha+2 p, \beta+\gamma+1-2 p)
\end{aligned}
$$

when

$$
y_{i} \neq y_{i+1}, x_{i} \neq x_{i+1}, \gamma+1=0,2,4,6, \ldots
$$

and

$$
Z(\gamma)= \begin{cases}0, & \gamma+1 \neq 2,4,6, \ldots \\ 1, & \gamma+1=2,4,6, \ldots \\ 0, & \gamma+1=0\end{cases}
$$

(iv)

$$
\begin{aligned}
I_{L}^{i, i+1}=y_{i, i+1} & \sum_{p=0}^{\gamma / 2} A_{i}(2 p, 2 p+1) F^{i, i+1}(\alpha+2 p+1, \beta+\gamma+1-2 p-1) \\
& +B_{i, i+1}(2 p, 2 p+1) H^{i, i+1}(\alpha+2 p+1, \beta+\gamma+1-2 p-1)
\end{aligned}
$$

when

$$
y_{i} \neq y_{i+1}, x_{i} \neq x_{i+1}, \gamma+1=1,3,5,7, \ldots
$$

with

$$
\begin{aligned}
\Phi_{j, \gamma+1-j}(l, m)= & \frac{l^{j} m^{\gamma+1-j}}{\lfloor j \mid \alpha+1-j} \frac{\lfloor\gamma+1}{(\alpha+1-j)}=\left(\begin{array}{c}
\gamma+1 \\
j
\end{array}\right) \frac{l^{j} m^{\gamma+1-j}}{(\alpha+j+1)}, \\
A_{i}(r, s)= & y_{i} \Phi_{r, \gamma+1-r}(l, m)+x_{i} \Phi_{s, \gamma+1-s}(l, m), \\
B_{i, i+1}(r, s)= & y_{i, i+1} \Phi_{r, \gamma+1-r}(l, m)+x_{i, i+1} \Phi_{s, \gamma+1-s}(l, m) \\
& \text { for } r, s=0,1,2, \ldots, \frac{\gamma+1}{2} \text { or } \frac{\gamma}{2}, \\
F^{i, i+1}(r, s)= & \int_{0}^{1} x^{r}(t) y^{s}(t) d t, \quad H^{i, i+1}(r, s)=\int_{0}^{1} t x^{r}(t) y^{s}(t) d t, \\
& x(t)=x_{i}+x_{i, i+1} t, \quad x_{i, i+1}=x_{i+1}-x_{i}, \\
& y(t)=y_{i}+y_{i, i+1} t, \quad y_{i, i+1}=y_{i+1}-y_{i} .
\end{aligned}
$$


LEMMA 4. The structure product $I I_{\pi}^{\alpha, \beta, \gamma}(l, m, h)$ (where $l \neq 0, m \neq 0$ and $h \neq 0$ are arbitrary real constants and $\alpha, \beta, \gamma$ are either zero or positive integers) over a simple polygon $\pi$ with $N$ oriented edges $l_{i, i+1}(i=1,2,3, \ldots, N)$ each with end points at $\left(x_{i}, y_{i}\right)$ and $\left(x_{i+1}, y_{i+1}\right)$ in the $x y$-plane is expressible as

$$
I I_{\pi}^{\alpha, \beta, \gamma}(l, m, h)=\iint_{\pi} x^{\alpha} y^{\beta}(l x+m y+h)^{\gamma+1} d x d y=\sum_{i=1}^{N} I_{L}^{i, i+1},
$$

where

(i)

$$
I_{L}^{i, i+1}=0, \quad \text { when } y_{i}=y_{i+1},
$$

(ii)

$$
\begin{aligned}
& I_{L}^{i, i+1}= y_{i, i+1} \sum_{j=0}^{\gamma+1} \sum_{r=0}^{j} \Phi_{r, j-r}(l, m, h) x_{i}^{\alpha+r+1} F^{i, i+1}(0, \beta+j-r) \\
& \text { when } y_{i} \neq y_{i+1}, x_{i}=x_{i+1},
\end{aligned}
$$

(iii)

$$
\begin{gathered}
I_{L}^{i, i+1}=y_{i, i+1}\left[\lambda_{i, i+1}^{0.1}+Z(\gamma) \sum_{j=2}^{\gamma+1} \lambda_{i, i+1}(\gamma)\right], \\
\text { when } y_{i} \neq y_{i+1}, \quad x_{i} \neq x_{i+1},
\end{gathered}
$$

with

$$
\begin{aligned}
\lambda_{i, i+1}^{0.1}= & P_{0} F(\alpha+1, \beta)+P_{1} H(\alpha+1, \beta), \\
\lambda_{i, i+1}(j)= & F^{i . i+1}(\alpha+1, \beta+j) \\
& +\sum_{p=1}^{j / 2} A_{j}(2 p-1,2 p) F^{i . i+1}(\alpha+2 p, \beta+j-2 p) \\
& \quad+B_{j}(2 p-1,2 p) H^{i . i+1}(\alpha+2 p, \beta+j-2 p) \\
& \text { for } j=2,4,6, \ldots,
\end{aligned}
$$




$$
\begin{aligned}
\lambda_{i, i+1}(j)= & \sum_{p=1}^{(j-1) / 2} A_{j}(2 p, 2 p+1) F^{i, i+1}(\alpha+2 p+1, \beta+j-2 p-1) \\
& +B_{j}(2 p, 2 p+1) H^{i, i+1}(\alpha+2 p+1, \beta+j-2 p-1),
\end{aligned}
$$

$$
\text { for } j=3,5,7, \ldots
$$

and

$$
\begin{aligned}
& Z(\gamma)= \begin{cases}0, & \gamma=0 \\
1, & \gamma>0\end{cases} \\
& F^{i, i+1}(r, s)=\int_{0}^{1} x^{r}(t) y^{s}(t) d t, \quad H^{i, i+1}(r, s)=\int_{0}^{1} t x^{r}(t) y^{s}(t) d t, \\
& x(t)=x_{i}+x_{i, i+1} t, \quad y(t)=y_{i}+y_{i, i+1} t, \\
& P_{0}=\Phi_{0.0}(l, m, h)+x_{i} \Phi_{1,0}(l, m, h)+y_{i} \Phi_{0.1}(l, m, h) \text {, } \\
& P_{1}=\Phi_{1,0}(l, m, h) x_{i, i+1}+\Phi_{0,1}(l, m, h) y_{i, i+1} \text {, } \\
& A_{j}(r, s)=y_{i} \Phi_{r, j-r}(l, m, h)+x_{i} \Phi_{s . j-s}(l, m, h), \\
& B_{j}(r, s)=y_{i, i+1} \Phi_{r, j-r}(l, m, h)+x_{i, i+1} \Phi_{s, j-s}(l, m, h) \\
& \text { for } j=2,3,4, \ldots, \gamma+1 \text {, } \\
& \Phi_{r, j-r}(l, m, h)=\frac{l^{r} m^{j-r} h^{\gamma+1-j}}{|r| j-r\lfloor\gamma+1-j} \frac{\lfloor\gamma+1}{(\alpha+r+1)}, \\
& 0 \leq j \leq \gamma+1,0 \leq r \leq j \text {. }
\end{aligned}
$$

LEMMA 5. The integrals defined as

$$
F^{i, i+1}(r, s)=\int_{0}^{1} x^{r}(t) y^{s}(t) d t \quad H^{i, i+1}(r, s)=\int_{0}^{1} t x^{r}(t) y^{s}(t) d t,
$$

wheré

$$
\begin{aligned}
& x(t)=x_{i}+x_{i, i+1} t, \quad y(t)=y_{i}+y_{i, i+1} t, \\
& x_{i, i+1}=x_{i+1}-x_{i}, \quad y_{i, i+1}=y_{i+1}-y_{i} \text {, }
\end{aligned}
$$

can be expressed as

$$
F^{i, i+1}(r, s)=\sum_{k=0}^{r+s} \frac{1}{k+1}\left(\sum_{\lambda=0}^{k} a_{r, \lambda}^{i . i+1} b_{s, k-\lambda}^{i, i+1}\right),
$$




$$
H^{i, i+1}(r, s)=\sum_{k=0}^{r+s} \frac{1}{k+2}\left(\sum_{\lambda=0}^{k} a_{r, \lambda}^{i, i+1} b_{s, k-\lambda}^{i, i+1}\right) \text {, }
$$

where

$$
a_{r, \lambda}^{i, i+1}=\left(\begin{array}{c}
r \\
\lambda
\end{array}\right) x_{i}^{r-\lambda} x_{i, i+1}^{\lambda} b_{s, k-\lambda}^{i, i+1}=\left(\begin{array}{c}
s \\
k-\lambda
\end{array}\right) y_{i}^{s-(k-\lambda)} y_{i, i+1}^{k-\lambda} .
$$

2.2. Consider the integral of (1) discussed in Lemmas $1,2,3$ and 4 , that is,

$$
I I_{\pi}^{\alpha . \beta . \gamma}(l, m, h)=\iint_{\pi} x^{\alpha} y^{\beta}(l x+m y+h)^{\gamma+1} d x d y,
$$

where $\pi$ is the simple polygon in the $x y$-plane as described in Figure $1, l, m, h$ are arbitrary constants and $\alpha, \beta, \gamma+1$ are either zero or positive integers. We can now think of $\pi$ as a region in $\mathbb{R}^{2}$ that may be decomposed into a set $T$ of triangles such that any pair of triangles $\tau_{i j k}^{x y}$ (a triangle in the $x y$-plane with vertices at $\left(x_{i}, y_{i}\right),\left(x_{j}, y_{j}\right)$ and $\left.\left(x_{k}, y_{k}\right)\right)$ and $\tau_{i^{\prime} j^{\prime} k^{\prime}}^{x y}$ do not intersect. The integral over the polygon $\pi$ can be split into integrals over the elements of a triangulation of $\pi$. Thus we may write

$$
I I_{\pi}^{\alpha, \beta . \gamma}(l, m, h)=\iint_{\pi} x^{\alpha} y^{\beta}(l x+m y+h)^{\gamma+1} d x d y=\sum_{\tau_{i j k}^{x y} \in T} I I_{\tau_{i j k}^{x y}}^{\alpha \beta \gamma}(l, m, h),(6
$$

where

$$
I I_{\tau_{i j k}^{* \gamma}}^{\alpha \beta \gamma}(l, m, h)=\iint_{\tau_{i j k}^{v j}} x^{\alpha} y^{\beta}(l x+m y+h)^{\gamma+1} d x d y
$$

LEMMA 6. The structure product $I_{\tau_{i j k}^{x y}}^{\alpha, \beta, \gamma}(l, m, h)$ over a triangle $\tau_{i j k}^{x y}$ in the $x y$-plane with vertices at $\left(x_{i}, y_{i}\right),\left(x_{j}, y_{j}\right)$ and $\left(x_{k}, y_{k}\right)$ can be expressed as

$$
I I_{\tau_{i j k}^{* y}}^{\alpha, \beta, \gamma}(l, m, h)=2 \Delta_{i j k} \sum_{n=0}^{\alpha+\beta+\gamma+1} \frac{S_{n}}{n+2},
$$

where

$$
\begin{aligned}
S_{n}= & \sum_{p=0}^{\frac{n}{2}-1}\lfloor p \mid n-p\{\lambda(p, n-p)+\lambda(n-p, p)\} \\
& +\left\lfloor\frac { n } { 2 } \left\lfloor\frac{n}{2} \lambda\left(\frac{n}{2}, \frac{n}{2}\right), \quad \text { for } n=2,4,6 \ldots,\right.\right. \\
S_{n}= & \sum_{p=0}^{\left(\frac{n-1}{2}\right)}\lfloor p \mid n-p\{\lambda(p, n-p)+\lambda(n-p, p)\} \quad \text { for } n=1,3,5 \ldots,
\end{aligned}
$$




$$
\begin{aligned}
S_{0}= & \lambda(0,0), \\
\lambda(p, q)= & \sum_{p_{1}=0}^{p} \sum_{p_{2}=0}^{p-p_{1}} \sum_{q_{1}=0}^{q} \sum_{q_{2}=0}^{q-q_{1}} F\left(p_{1}, q_{1}\right) G\left(p_{2}, q_{2}\right) H\left(p_{3}, q_{3}\right), \\
& p+q=n, \quad p_{3}=p-p_{1}-p_{2}, \quad q_{3}=q-q_{1}-q_{2},
\end{aligned}
$$

$$
\begin{aligned}
& F\left(p_{1}, q_{1}\right)=\left\lfloor\alpha \frac{a_{x}^{p_{1}} b_{x}^{q_{1}} x_{i}^{\alpha-\left(p_{1}+q_{1}\right)}}{\left\lfloorp _ { 1 } \left\lfloorq _ { 1 } \left\lfloor\alpha-\left(p_{1}+q_{1}\right)\right.\right.\right.},\right. \\
& G\left(p_{2}, q_{2}\right)=\left\lfloor\frac{a_{y}^{p_{2}} b_{y}^{q_{2}} y_{i}^{\beta-\left(p_{2}+q_{2}\right)}}{\left\lfloorp _ { 2 } \left\lfloorq _ { 2 } \left\lfloor\beta-\left(p_{2}+q_{2}\right)\right.\right.\right.},\right. \\
& H\left(p_{3}, q_{3}\right)=\left\lfloor\gamma+1 \frac{a_{z}^{p_{3}} b_{z}^{q_{3}} z_{i}^{\gamma+1-\left(p_{3}+q_{3}\right)}}{\left\lfloorp _ { 3 } \left\lfloorq _ { 3 } \left\lfloor\gamma+1-\left(p_{3}+q_{3}\right)\right.\right.\right.},\right.
\end{aligned}
$$

$$
\begin{aligned}
\Delta_{i j k} & =\text { area of triangle with vertices at }\left(x_{i}, y_{i}\right),\left(x_{j}, y_{j}\right) \text { and }\left(x_{k}, y_{k}\right) \\
& =\frac{1}{2}\left(a_{x} b_{y}-b_{x} a_{y}\right)=\frac{1}{2}\left|\begin{array}{ccc}
1 & 1 & 1 \\
x_{i} & x_{j} & x_{k} \\
y_{i} & y_{j} & y_{k}
\end{array}\right|
\end{aligned}
$$

PROOF. The parametric vectorial equation of the oriented triangle $\tau_{i j k}^{x y}$ in the $x y$-plane with vertices at $\left(x_{i}, y_{i}\right),\left(x_{j}, y_{j}\right)$ and $\left(x_{k}, y_{k}\right)$ is

$$
\bar{p}=\mathbf{p}_{i}+\mathbf{a} u+\mathbf{b} v, \quad 0 \leq u, v \leq 1, u+v \leq 1,
$$

where

$$
\mathbf{p}=(x, y), \quad \mathbf{a}=\mathbf{p}_{j}-\mathbf{p}_{i}, \quad \mathbf{b}=\mathbf{p}_{k}-\mathbf{p}_{i}, \quad \mathbf{a}=\left(a_{x}, x_{y}\right), \quad \mathbf{b}=\left(b_{x}, b_{y}\right) .
$$

If we now consider the mapping (see Figure 2) between the $x y$-plane and the $u v$-plane defined in (65), we have for the area element

$$
\begin{aligned}
d x d y & =|J| d u d v, \\
|J| & =\operatorname{det} J=\frac{\partial x}{\partial u} \cdot \frac{\partial y}{\partial v}-\frac{\partial x}{\partial v} \cdot \frac{\partial y}{\partial u}=a_{x} b_{y}-b_{x} a_{y}=2 \Delta_{i, j, k} \\
& =2 \times \text { area of triangle } \tau_{i j k}^{x y},
\end{aligned}
$$

where $J$ is the Jacobian of the transformation.

If we change the coordinates according to (64) and express the area element using (66) we obtain

$$
I I_{\tau_{i j k}^{\alpha \beta}}^{\alpha, \beta, \gamma}(l, m, h)
$$



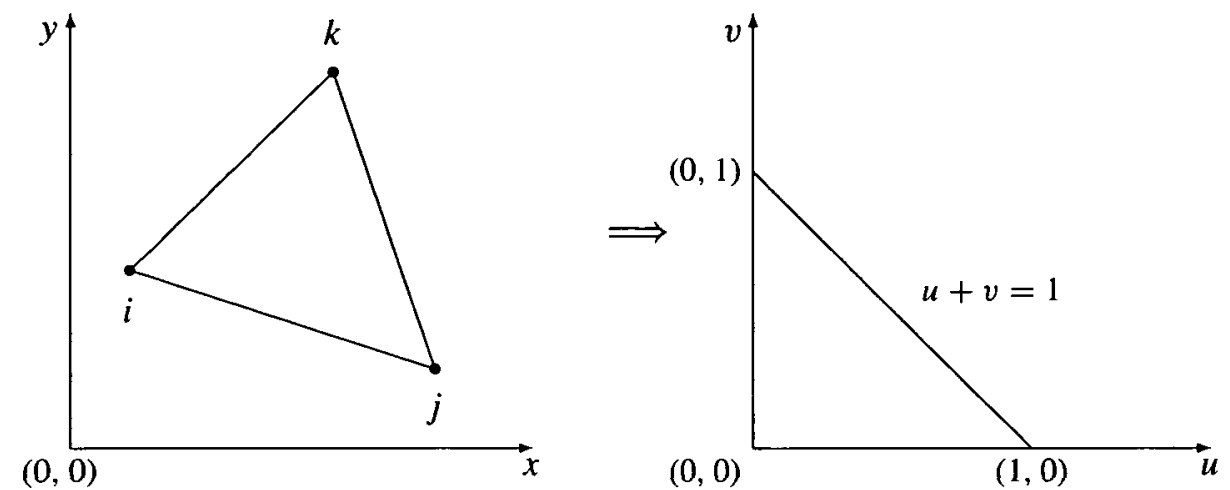

FIGURE 2. The mapping between an oriented triangle in the $x y$-plane and the unit triangle in the $u v$-plane.

$$
\begin{aligned}
& =\iint_{\tau_{i j k}^{x k}} x^{\alpha} y^{\beta}(l x+m y+h)^{\gamma+1} d x d y \\
& =\iint_{i}\left[x_{i}+a_{x} u+b_{x} v\right]^{\alpha}\left[y_{i}+a_{y} u+b_{y} v\right]^{\beta}\left[z_{i}+a_{z} u+b_{z} v\right]^{\gamma+1}\left(2 \Delta_{i j k}\right) d u d v
\end{aligned}
$$

where

$$
\begin{aligned}
z(u, v) & =l x(u, v)+m y(u, v)+h=z_{i}+a_{z} u+b_{z} v \\
z_{i} & =l x_{i}+m y_{i}+h, \quad a_{z}=z_{j}-z_{i}, \quad b_{z}=z_{k}-z_{i}, \\
z_{j} & =l x_{j}+m y_{j}+h, \quad z_{k}=l x_{k}+m y_{k}+h
\end{aligned}
$$

and $\bar{\tau}$ is the unit triangle spanning the vertices $(0,0),(1,0)$ and $(0,1)$ in the $u v$-plane.

We can now rewrite (67) as

$$
I I_{\tau_{i j k}^{*, \beta \gamma}}^{\alpha, \beta}(l, m, h)=\iint_{\bar{\tau}} f(u, v)\left(2 \Delta_{i j k}\right) d u d v
$$

where

$$
\begin{aligned}
& f(u, v)=X(u, v) Y(u, v) Z(u, v), \\
& X(u, v)=\left(x_{i}+a_{x} u+b_{x} v\right)^{\alpha} \\
& Y(u, v)=\left(y_{i}+a_{y} u+b_{y} v\right)^{\beta} \\
& Z(u, v)=\left(z_{i}+a_{z} u+b_{z} v\right)^{\gamma+1} .
\end{aligned}
$$

We use Taylor's theorem to expand each of the functions $f(u, v), X(u, v), Y(u, v)$ and $Z(u, v)$ in powers of $u$ and $v$ so that we may write

$$
X(u, v)=\sum_{j=0}^{\alpha} \frac{1}{\lfloor j}\left(u \frac{\partial}{\partial u}+v \frac{\partial}{\partial v}\right)_{(0.0)}^{j} X(u, v) .
$$


We now apply the binomial theorem to expand (71) to obtain

$$
\begin{aligned}
X(u, v) & =\sum_{j=0}^{\alpha} \sum_{r=0}^{j} \frac{u^{r} v^{s}}{r}\left\lfloor^{s}\left(\frac{\partial^{j} X(u, v)}{\partial u^{r} \partial v^{s}}\right)_{(0.0)}\right. \\
& =\sum_{j=0}^{\alpha} \sum_{r=0}^{j}\left\lfloor\frac{a_{x}^{r} b_{x}^{s} x_{i}^{\alpha-j}}{r} u^{r} v^{s-j} \quad(\text { where } s=j-r) .\right.
\end{aligned}
$$

In a similar manner, we can derive

$$
\begin{aligned}
& Y(u, v)=\sum_{j=0}^{\beta} \sum_{r=0}^{j} \frac{\beta}{\frac{a_{y}^{r} b_{y}^{s} y_{i}^{\beta-j}}{r} \bigsqcup^{s} \bigsqcup^{\beta-j}} u^{r} v^{s}, \\
& Z(u, v)=\sum_{j=0}^{\gamma+1} \sum_{r=0}^{j} l \gamma+1 \frac{a_{z}^{r} b_{z}^{s} z_{i}^{\gamma+1-j}}{r \leq \underline{r}\lfloor\gamma+1-j} u^{r} v^{s}, \\
& f(u, v)=\sum_{n=0}^{\alpha+\beta+\gamma+1} \sum_{p=0}^{n} \frac{1}{p}\left\{\frac{\partial^{n} f(u, v)}{\partial u^{p} \partial v^{q}}\right\}_{(0,0)} u^{p} v^{q} \text {, }
\end{aligned}
$$

where $q=n-p$. have

We determine $\left\{\frac{\partial^{n} f(u, v)}{\partial u^{p} \partial d v^{q}}\right\}_{(0,0)}$ by using Leibnitz's theorem on differentiation. We

$$
\begin{aligned}
& \frac{\partial^{p} f(u, v)}{\partial u^{p}} \\
& \quad=\sum_{p_{1}=0}^{p}\left(\begin{array}{c}
p \\
p_{1}
\end{array}\right)\left\{\frac{\partial^{p_{1}} X(u, v)}{\partial u^{p_{1}}}\right\}\left\{\frac{\partial^{p-p_{1}}[Y(u, v) Z(u, v)]}{\partial u^{p-p_{1}}}\right\} \\
& =\sum_{p_{1}=0}^{p}\left(\begin{array}{c}
p \\
p_{1}
\end{array}\right) \frac{\partial^{p_{1}} X(u, v)}{\partial u^{p_{1}}} \sum_{p_{2}=0}^{p-p_{1}}\left(\begin{array}{c}
p-p_{1} \\
p_{2}
\end{array}\right) \frac{\partial^{p_{2}} Y(u, v)}{\partial u^{p_{2}}} \frac{\partial^{p-p_{1}-p_{2}} Z(u, v)}{\partial u^{p-p_{1}-p_{2}}} \\
& \quad=\sum_{p_{1}=0}^{p} \sum_{p_{2}=0}^{p-p_{1}} \frac{\lfloor p}{p_{1}} \frac{\partial^{p_{2}}}{p-p_{1}-p_{2}} \frac{\partial^{p-v}(u, v)}{\partial u^{p_{1}}} \frac{\partial^{p_{2}} Y(u, v)}{\partial u^{p_{2}}} \frac{\partial^{p-p_{1}-p_{2}} Z(u, v)}{\partial u^{p-p_{1}-p_{2}}} .
\end{aligned}
$$

Differentiating of (76) $q$ times with respect to $v$ and using Leibnitz's theorem we obtain

$$
\left[\frac{\partial^{p+q} f(u, v)}{\partial u^{p} \partial v^{q}}\right]_{(0.0)} /\left(\left\lfloor^{p}, q\right),\right.
$$

that is,

$$
\left[\frac{\partial^{p+q} f(u, v)}{\partial u^{p} \partial v^{q}}\right]_{(0,0)} /(\lfloor p\lfloor)
$$




$$
\begin{aligned}
& =\sum_{p_{1}=0}^{p} \sum_{p_{2}=0}^{p-p_{1}} \frac{1}{\left\lfloorp _ { 1 } \left\lfloorp _ { 2 } \left\lfloor p_{3}\right.\right.\right.} \sum_{q_{1}=0}^{q} \sum_{q_{2}=0}^{q-q_{1}} \frac{1}{\left\lfloorq _ { 1 } \left\lfloorq _ { 2 } \left\lfloor q_{3}\right.\right.\right.}\left[\frac{\partial^{p_{1}+q_{1}} X(u, v)}{\partial u^{p_{1}} \partial v^{q_{1}}}\right]_{(0,0)} \\
& \times\left[\frac{\partial^{p_{2}+q_{2}} Y(u, v)}{\partial u^{p_{2}} \partial v^{q_{2}}}\right]_{(0,0)}\left[\frac{\partial^{p_{3}+q_{3}} Z(u, v)}{\partial u^{p_{3}} \partial v^{q_{3}}}\right]_{(0,0)},
\end{aligned}
$$

where

$$
p_{1}+p_{2}+p_{3}=p, \quad q_{1}+q_{2}+q_{3}=q \text {. }
$$

We also have from (72), (73) and (74) that

$$
\begin{aligned}
& {\left[\frac{\partial^{p_{1}+q_{1}} X(u, v)}{\partial u^{p_{1}} \partial v^{q_{1}}}\right]_{(0,0)} /\left(\mid p_{1}\left\lfloor q_{1}\right)=F\left(p_{1}, q_{1}\right)\right. \text { (say) }} \\
& =\left\lfloor\alpha \frac{a_{x}^{p_{1}} b_{x}^{q_{1}} x_{i}^{\alpha-\left(p_{1}+q_{1}\right)}}{\left\lfloorp _ { 1 } \left\lfloorq _ { 1 } \left\lfloor-\left(p_{1}+q_{1}\right)\right.\right.\right.}\right. \\
& {\left[\frac{\partial^{p_{1}+p_{2}} Y(u, v)}{\partial u^{p_{2}} \partial v^{q_{2}}}\right]_{(0,0)} /\left(\left(p_{2}\left\lfloor q_{2}\right)=G\left(p_{2}, q_{2}\right)\right.\right. \text { (say) }} \\
& =\beta \frac{a_{y}^{p_{2}} b_{y}^{q_{2}} y_{i}^{\beta-\left(p_{2}+q_{2}\right)}}{p_{2}\left|q_{2}\right| \beta-\left(p_{2}+q_{2}\right)}, \\
& {\left[\frac{\partial^{p_{3}+q_{3}} Z(u, v)}{\partial u^{p_{3}} \partial v^{q_{3}}}\right]_{(0,0)} /\left(\left\lfloorp_{3}\left\lfloor q_{3}\right)=H\left(p_{3}, q_{3}\right)\right.\right. \text { (say) }} \\
& =\left\lfloor\gamma+1 \frac{a_{z}^{p_{3}} b_{z}^{q_{3}} z_{i}^{\gamma+1-\left(p_{3}+q_{3}\right)}}{\left\lfloorp _ { 3 } \left\lfloorq _ { 3 } \left\lfloor\gamma+1-\left(p_{3}+q_{3}\right)\right.\right.\right.} .\right.
\end{aligned}
$$

From (77) - (81), we can write

$$
\begin{aligned}
{\left[\frac{\partial^{p+q} f(u, v)}{\partial u^{p} \partial v^{q}}\right]_{(0,0)} /(\lfloor p\lfloor q)} & =\lambda(p, q) \quad(\text { say }) \\
& =\sum_{p_{1}=0}^{p} \sum_{p_{2}=0}^{p-p_{1}} \sum_{q_{1}=0}^{q} \sum_{q_{2}=0}^{q-q_{1}} F\left(p_{1}, q_{1}\right) G\left(p_{2}, q_{2}\right) H\left(p_{3}, q_{3}\right)
\end{aligned}
$$

Finally using (75) and (82), we can write

$$
f(u, v)=\sum_{n=0}^{\alpha+\beta+\gamma+1} \sum_{p=0}^{n} \lambda(p, q) u^{p} v^{q}, \quad q=n-p .
$$


Substituting for $f(u, v)$ from (83) into (69) we obtain

$$
\begin{aligned}
I I_{\tau_{i j k}^{*}, \beta, \gamma}^{\alpha, \beta,}(l, m, h) & =2 \Delta_{i j k} \iint_{\bar{\tau}} f(u, v) d u d v \\
& =2 \Delta_{i j k} \iint_{\bar{i}}^{\alpha+\beta+\gamma+1} \sum_{n=0}^{n} \sum_{p=0}^{n} \lambda(p, n-p) u^{p} v^{n-p} d u d v \\
& =2 \Delta_{i j k} \sum_{n=0}^{\alpha+\beta+\gamma+1} \frac{S_{n}}{n+2},
\end{aligned}
$$

where

$$
S_{n}=\sum_{p=0}^{n} \underline{n-p} \lambda(p, n-p) .
$$

Clearly

$$
S_{0}=\lambda(0,0) \text {. }
$$

We now determine a simple expression for $S_{n}(n=1,2,3, \ldots, \alpha+\beta+\gamma+1)$.

(i) Let $n=2,4,6, \ldots$ Then from (85)

$$
\begin{aligned}
S_{n} & =\sum_{p=0}^{n} \lambda(p, n-p)\lfloor\underline{\underline{p}} \underline{\underline{n}-p} \\
& =\sum_{p=0}^{n / 2-1} \underline{\underline{p}\lfloor n-p}\{\lambda(p, n-p)+\lambda(n-p, p)\}+\left\lfloor\frac { n } { 2 } \left\lfloor\frac{n}{2} \lambda\left(\frac{n}{2}, \frac{n}{2}\right) .\right.\right.
\end{aligned}
$$

(ii) Let $n=1,3,5,7, \ldots$ Then from (85)

$$
\begin{aligned}
S_{n} & =\sum_{p=0}^{n} \lambda(p, n-p)\lfloor p\lfloor n-p \\
& =\sum_{p=0}^{\left(\frac{n-1}{2}\right)}\lfloor p-p\{\lambda(p, n-p)+\lambda(n-p, p)\} .
\end{aligned}
$$

The results obtained in (84) through (88) prove Lemma 6.

\section{Volume integration}

Let $V$ be a three-dimensional polyhedron bounded by a polyhedral surface $\partial V$. Then the volume integral of a scalar function $f(\mathbf{p})=x^{\alpha} y^{\beta} z^{\gamma}(\alpha, \beta, \gamma$ are either zero or positive integers) can be easily derived by using Gauss's divergence theorem. 
THEOREM A. A structure product

$$
I I I_{V}^{\alpha . \beta . \gamma}=\iiint_{V} x^{\alpha} y^{\beta} z^{\gamma} d V
$$

over a linear polyhedron $V$ is a polynomial combination of structure products of suitable order over a triangulation of the polyhedral boundary $\partial V$ and it is expressible as

$$
\begin{aligned}
I I I_{V}^{\alpha . \beta . \gamma} & =\sum_{\tau_{i j, j \in T} \in T} \frac{1}{(\gamma+1)} \iint_{\tau_{i j k}} x^{\alpha} y^{\beta} z^{\gamma+1} \mathbf{k} \cdot \mathbf{n} d \tau \\
& =\sum_{\tau_{i j k}^{y *} \in T} \frac{1}{(\gamma+1)} \iint_{\tau_{i j k}^{x}} x^{\alpha} y^{\beta}(l x+m y+h)^{\gamma+1} K d x d y,
\end{aligned}
$$

where

(i) $T$ is a triangulation of the polyhedral surface $\partial V$,

(ii) $\tau_{i j k} \in T$ is a linear triangle in the three-dimensional space with vertices at $\left(x_{i}, y_{i}, z_{i}\right),\left(x_{j}, y_{j}, z_{j}\right) ;$ and $\left(x_{k}, y_{k}, z_{k}\right), \mathbf{n}$ is the outward unit normal vector to $\tau_{i j k}$ and $\mathbf{k}$ is the unit normal vector along the $z$-axis,

(iii) $z=l x+m y+h,(l, m, h$ are arbitrary constants) is the equation of the plane spanning the three vertices $\left(x_{i}, y_{i}, z_{i}\right),\left(x_{j}, y_{j}, z_{j}\right)$ and $\left(x_{k}, y_{k}, z_{k}\right)$ of a linear triangle $\tau_{i j k} \in T$,

(iv) $\tau_{i j k}^{x y}$ is a projection of a linear triangle $\tau_{i j k}$ in three-dimensional space onto the $x y$-plane with vertices at $\left(x_{i}, y_{i}\right),\left(x_{j}, y_{j}\right)$ and $\left(x_{k}, y_{k}\right)$,

(v) $K=0,+1,-1$, depends on the orientation of the linear triangle $\tau_{i j k}$ in three-dimensional space.

PROOF. Let $V$ be a three-dimensional polyhedron bounded by a polyhedral surface. Let $\mathbf{F}=\left(0,0, x^{\alpha} y^{\beta} z^{\gamma+1} /(\gamma+1)\right)$ be a vector field in three-dimension space. Then $x^{\alpha} y^{\beta} z^{\gamma}=\nabla \cdot \mathbf{F}$ and if we assume regularity of the integration domain and continuity of the integrand, we have from Gauss's divergence theorem that

$$
\begin{aligned}
\iiint_{V} x^{\alpha} y^{\beta} z^{\gamma} d x d y d z & =I I I_{V}^{\alpha, \beta, \gamma}=\iiint_{V} \boldsymbol{\nabla} \cdot \mathbf{F} d x d y d z=\iint_{\partial V} \mathbf{F} \cdot \mathbf{n} d S \\
& =\frac{1}{(\gamma+1)} \iint_{\partial V} x^{\alpha} y^{\beta} z^{\gamma+1} \mathbf{k} \cdot \mathbf{n} d S \\
& =\frac{1}{(\gamma+1)} \sum_{\tau_{i j k} \in T} x^{\alpha} y^{\beta} z^{\gamma+1} \mathbf{k} \cdot \mathbf{n} d \tau .
\end{aligned}
$$

The results of (92) and (93) follow from the fact that $F_{x}=0, F_{y}=0$ which gives a null contribution to the scalar product inside the integral sign. This establishes (90). We now proceed to prove (91). 
The equation of the plane for the linear triangle $\tau_{i j k}$ spanning the vertices $\left\{\left(x_{i}, y_{i}, z_{i}\right)\right.$, $\left.\left(x_{j}, y_{j}, z_{j}\right),\left(x_{k}, y_{k}, z_{k}\right)\right\}$ is given by

$$
\left(x-x_{i}\right) J_{i j k}(y, z)+\left(y-y_{i}\right) J_{i j k}(z, x)+\left(z-z_{i}\right) J_{i j k}(x, y)=0,
$$

where

$$
\begin{aligned}
& J_{i j k}(y, z)=\left(y_{j}-y_{i}\right)\left(z_{k}-z_{i}\right)-\left(z_{j}-z_{i}\right)\left(y_{k}-y_{i}\right), \\
& J_{i j k}(z, x)=\left(z_{j}-z_{i}\right)\left(x_{k}-x_{i}\right)-\left(x_{j}-x_{i}\right)\left(z_{k}-z_{i}\right), \\
& J_{i j k}(x, y)=\left(x_{j}-x_{i}\right)\left(y_{k}-y_{i}\right)-\left(y_{j}-y_{i}\right)\left(x_{k}-x_{i}\right) .
\end{aligned}
$$

CASE 1. If $J_{i j k}(x, y) \neq 0, J_{i j k}(y, z) \neq 0, J_{i j k}(z, x) \neq 0$, we can write (94) as

$$
z=l x+m y+h
$$

where

$$
\begin{aligned}
& l=-J_{i j k}(y, z) / J_{i j k}(x, y), \quad m=-J_{i j k}(z, x) / J_{i j k}(x, y), \\
& h=\left\{x_{i} J_{i j k}(y, z)+y_{i} J_{i j k}(z, x)+z_{i} J_{i j k}(x, y)\right\} / J_{i j k}(x, y) .
\end{aligned}
$$

Let us now analyse the surface integral

$$
I I I_{\tau_{i j k}}^{\alpha, \beta, \gamma}=\iint_{\tau_{i j k}} x^{\alpha} y^{\beta} z^{\gamma+1} \mathbf{k} \cdot \mathbf{n} d \tau
$$

Using definition (93) as

$$
I I I_{V}^{\alpha, \beta, \gamma}=\iiint_{V} x^{\alpha} y^{\beta} z^{\gamma} d x d y d z=\frac{1}{(\gamma+1)} \sum_{\tau_{i j k} \in T} I I I_{\tau_{i j k}}^{\alpha, \beta, \gamma},
$$

we know from the theory of surface integrals that if $z=f(x, y)$ is the equation of the surface then we can write

$$
\begin{aligned}
d \tau & =\left\{1+\left(\frac{\partial f}{\partial x}\right)^{2}\left(\frac{\partial f}{\partial y}\right)^{2}\right\}^{1 / 2} d x d y \\
\mathbf{n} & = \pm\left(-\frac{\partial f}{\partial x} \mathbf{i}-\frac{\partial f}{\partial y} \mathbf{j}+\mathbf{k}\right) /\left\{1+\left(\frac{\partial f}{\partial x}\right)^{2}\left(\frac{\partial f}{\partial y}\right)^{2}\right\}^{1 / 2}
\end{aligned}
$$

Thus from (98) and (99), we find that

$$
\mathbf{k} \cdot \mathbf{n} d \tau= \pm d x d y
$$


and from (96), we also have that

$$
z=f(x, y)=l x+m y+h .
$$

Using (102) and (103) we can write (98) as

$$
I I I_{\tau_{i j k}}^{\alpha, \beta . \gamma}=\iint_{\tau_{i j k}} x^{\alpha} y^{\beta} z^{\gamma+1} \mathbf{k} \cdot \mathbf{n} d \tau= \pm \iint_{\tau_{i j k}^{r v}} x^{\alpha} y^{\beta}(l x+m y+h)^{\gamma+1} d x d y .
$$

In (104), we have to choose the positive sign if $J_{i j k}(x, y)>0$, which corresponds to positive orientation of the projection $\tau_{i j k}^{x y}$. The contrary sign simply means that the projection is in a clockwise orientation.

CASE 2. If $J_{i j k}(y, z)=0, J_{i j k}(z, x)=0$ and $J_{i j k}(x, y) \neq 0$, then the equation of the plane in (94) reduces to $z=z_{i}$, so that we may take $l=0 m=0$ and $h=z_{i}$ in (103). So from (104)

$$
I I I_{\tau_{i j k}}^{\alpha, \beta, \gamma}= \pm \iint_{\tau_{i j k}^{\gamma}} x^{\alpha} y^{\beta}\left(z_{i}\right)^{\gamma+1} d x d y
$$

Clearly, from (105),

$$
I I I_{\tau_{i j k}}^{\alpha, \beta . \gamma}=0, \quad \text { if } \quad z_{i}=0 .
$$

We have to choose the positive sign in (105) when the plane $z=z_{i}$ is bounded by planes $z=z_{i}-\epsilon$ for arbitrarily small $\epsilon>0$ contained in $V$. We have to choose the negative sign in (105) when the plane $z=z_{i}$ is bounded above by planes $z=z_{i}+\epsilon$ for arbitrarily small $\epsilon>0$ contained in $V$.

CASE 3. If $J_{i j k}(x, y)=0$ and either or both of $J_{i j k}(y, z)$ and $\tau_{i j k}(z, x)$ are nonzero, from (94) the equation of the plane containing the linear triangle $\tau_{i j k}$ is given by

$$
\left(x-x_{i}\right) J_{i j k}(y, z)+\left(y-y_{i}\right) J_{i j k}(z, x)=0 .
$$

From (107), we have

$$
\mathbf{n}= \pm \frac{\nabla\left\{\left(x-x_{i}\right) J_{i j k}(y, z)+\left(y-y_{i}\right) J_{i j k}(z, x)\right\}}{\left|\nabla\left\{\left(x-x_{i}\right) J_{i j k}(y, z)+\left(y-y_{i}\right) J_{i j k}(z, x)\right\}\right|} .
$$

From (108) we find

$$
\mathbf{n} \cdot \mathbf{k}=0
$$

and from (108), we obtain

$$
I I I_{\tau_{i j k}}^{\alpha, \beta, \gamma}=\iint_{\tau_{i j k}} x^{\alpha} y^{\beta} z^{\gamma+1} \mathbf{k} \cdot \mathbf{n} d \tau=0 .
$$

The results obtained in (104), (105), (106) and (110) prove the theorem. 


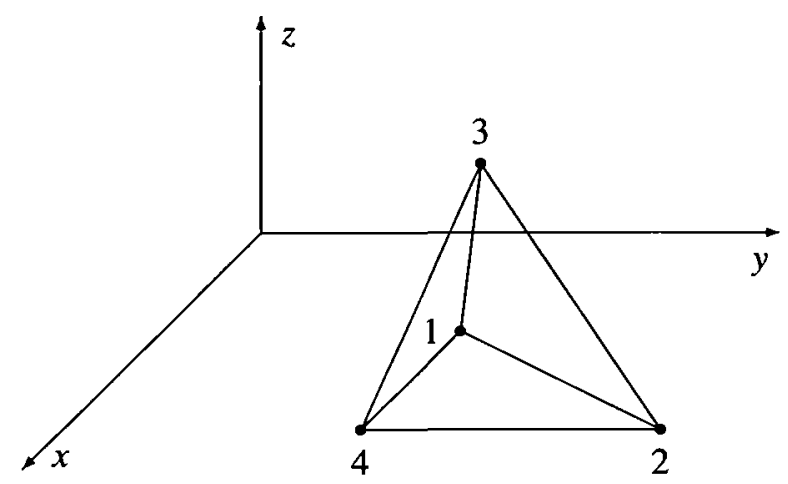

FIGURE 3. A tetrahedron with vertices $V_{i}(i=1,2,3,4)$.

\section{Application - Example}

Consider the evaluation of volume integral $\iiint_{V} x^{2} y d x d y d z$, where $V$ is a tetrahedron bounded by tetrahedral surface $\partial V$ with vertices $V_{i}$ as shown in Figure 3 .

$$
\begin{aligned}
& V_{1}=\left(x_{1}, y_{1}, z_{1}\right)=(5,5,0) . \\
& V_{2}=\left(x_{2}, y_{2}, z_{2}\right)=(10,10,0) . \\
& V_{3}=\left(x_{3}, y_{3}, z_{3}\right)=(8,7,8) . \\
& V_{4}=\left(x_{4}, y_{4}, z_{4}\right)=(10,5,0) .
\end{aligned}
$$

By using the theorem of the previous section, we can write

$$
\iiint_{V} x^{2} y d x d y d z=\iint_{S} x^{2} y z \mathbf{k} \cdot \mathbf{n} d S
$$

where $S$ is the surface of the tetrahedron consisting of linear triangles (in $\mathbb{R}^{3}$ ) $\tau_{421}, \tau_{234}$, $\tau_{123}, \tau_{431}$ and $\mathbf{n}$ is the outward unit normal vector to $S$ (see Figure 3). Hence using (90)

$$
\begin{aligned}
\iiint_{V} x^{2} y d x d y d z & =\sum_{\tau_{i j k} \in S} \iint_{\tau_{i j k}} x^{2} y z \mathbf{k} \cdot \mathbf{n} d \tau \\
& =\left(\iint_{\tau_{421}}+\iint_{\tau_{2,4}}+\iint_{\tau_{123}}+\iint_{\tau_{431}}\right) x^{2} y z \mathbf{k} \cdot \mathbf{n} d \tau .
\end{aligned}
$$

We can evaluate each of the above integrals on the right-hand side of (112) by using the theorem of the previous section. By (91) we obtain

$$
\iiint_{V} x^{2} y d x d y d z=\iint_{\tau_{24}^{x V}} x^{2} y\{-4(x-10)\} d x d y-\iint_{\tau_{123}^{x y}} x^{2} y\{8(x-y)\} d x d y
$$




$$
+\iint_{\mathbf{x}_{431}^{x y}} x^{2} y\{4(y-5)\} d x d y .
$$

We can evaluate the integrals of (113) by using Lemmas 1, 2, 3, 4, 5 or Lemma 6. We shall illustrate this in Algorithms 1 and 2 in the succeeding pages.

4.1. Algorithm 1 We shall now illustrate first the use of Lemmas 1 to 5 to compute integrals of the type

$$
I I_{\tau_{i j k}^{x y}}^{\alpha, \beta, \gamma}(l, m, h)=\iint_{\tau_{i j k}^{x y}} x^{\alpha} y^{\beta}(l x+m y+h)^{\gamma+1} d x d y .
$$

With (112), we have $\alpha=2, \beta=1, \gamma=0$. Let us first consider the computation of the integral $\iint_{r_{234}^{x y}} x^{2} y(-4 x+40) d x d y$.

We have using Lemma 2

$$
\iint_{\tau_{234}^{x y}} x^{2} y(-4 x+40) d x d y=-\sum_{(p, q)} \sum_{P \in\{(2,3),(3,4)\}} I_{L}^{p, q},
$$

where

$$
I_{L}^{p, q}=\int_{l_{p q}} \frac{x^{2} y^{2}}{2}(-4 x+40) d x .
$$

We have on using Lemma 2 again that

$$
I_{L}^{p, q}=\frac{x_{p, q}}{2} \sum_{j=0}^{5} \mu_{p q}(j) /(j+1)
$$

where

$$
\begin{gathered}
\mu_{p . q}(j)=\sum_{j_{1}=0}^{j} \sum_{j_{2}=0}^{j-j_{1}} F_{p q}\left(2-j_{1}, j_{1}\right) G_{p q}\left(2-j_{2}, j_{2}\right) H_{p q}\left(1-j+j_{1}+j_{2}, j-j_{1}-j_{2}\right), \\
F_{p q}(2,0)=x_{p}^{2}, \quad F_{p q}(1,1)=2 x_{p} x_{p q}, \quad F_{p q}(0,2)=x_{p q}^{2} \\
G_{p q}(2,0)=y_{p}^{2}, \quad G_{p q}(1,1)=2 y_{p} y_{p q}, \quad G_{p q}(0,2)=y_{p q}^{2}, \\
H_{p q}(1,0)=z_{p}, \quad H_{p q}(0,1)=z_{p q} .
\end{gathered}
$$

We now show the details of the computation for $(p, q)=(2,3)$. For the other pair $(p, q)=(3,4)$ the computational procedure is similar.

Choosing

$$
\begin{aligned}
p & =2, & q & =3, & l & =-4, \quad h=40, \\
x_{2} & =10, & y_{2} & =10, & z_{2} & =l x_{2}+h=0, \\
x_{3} & =8, & y_{3} & =7, & z_{3} & =l x^{3}+h=8 \\
x_{23} & =-2, & y_{23} & =-3, & z_{23} & =8
\end{aligned}
$$


and substituting from (119) into (118), we obtain from (117) that

$$
\begin{aligned}
I_{L}^{2.3} & =\int_{l_{23}} \frac{x^{2} y^{2}}{2}(-4 x+40) d x \\
& =\frac{x_{23}}{2}\left\{\mu_{23}(0)+\frac{\mu_{23}(1)}{2}+\frac{\mu_{23}(2)}{3}+\frac{\mu_{23}(3)}{4}++\frac{\mu_{23}(4)}{5}+\frac{\mu_{23}(5)}{6}\right\} \\
& =\frac{(-2)}{2}\left\{0+\frac{80000}{2}+\frac{(-80,000)}{3}+\frac{(29600)}{4}+\frac{(-4800)}{5}+\frac{288}{6}\right\} \\
& =\frac{-59464}{3} .
\end{aligned}
$$

In a similar manner, we obtain

$$
I_{L}^{3.4}=\iint_{l_{34}} \frac{x^{2} y^{2}}{2}(-4 x+40) d x=\frac{35880}{3}
$$

from (115), (120) and (121), we obtain

$$
\iint_{\tau_{234}} x^{2} y(-4 x+40) d x d y=\frac{23584}{3} \text {. }
$$

Now using Lemma 1 we obtain

$$
\iint_{\mathfrak{\tau}_{431}^{x y}} x^{2} y\{4 y-20\} d x d y=\sum_{(p, q) \in\{(4.3) .(3,1)\}} I_{L}^{p \cdot q}
$$

where

$$
I_{L}^{p q}=\int_{l_{p q}} \frac{x^{3} y}{3}(4 y-20) d y
$$

Using Lemma 1 again,

$$
I_{L}^{p . q}=\frac{y_{p q}}{3} \sum_{j=0}^{5} \lambda_{p q}(j) /(j+1)
$$

where

$$
\begin{aligned}
& \lambda_{p q}(j)=\sum_{j_{1}=0}^{j} \sum_{j_{2}=0}^{j-j_{1}} F_{p q}\left(3-j_{1}, j_{1}\right) H_{p q}\left(1-j_{2}, j_{2}\right) H_{p q}\left(1-j+j_{1}+j_{2}, j-j_{1}-j_{2}\right) \\
& F_{p q}(3,0)=x_{p}^{3}, F_{p q}(2,1)=3 x_{p}^{2} x_{p q}, \quad F_{p q}(1,2)=3 x_{p} x_{p q}^{2} \\
& F_{p q}(0,3)=x_{p q}^{3}, G_{p q}(1,0)=y_{p}, \\
& H_{p q}(1,0)=z_{p}, H_{p q}(0,1)=z_{p q} .
\end{aligned}
$$


We now show the details of computation for $(p, q)=(4,3)$. For the other pair, the computational procedure is similar.

Choosing

$$
\begin{aligned}
& p=4, \quad q=3, \quad l=0, \quad m=4, \quad h=-20, \\
& x_{4}=10, \quad y_{4}=5, \quad z_{4}=m y_{4}+h=0, \\
& x_{3}=8, \quad y_{3}=7, \quad z_{3}=m y_{3}+h=8, \\
& x_{43}=-2, \quad y_{43}=2, \quad z_{43}=8
\end{aligned}
$$

and substituting (127) into (126), we obtain from (125)

$$
\begin{aligned}
I_{L}^{4.3} & =\frac{y_{43}}{3} \sum_{j=0}^{5} \lambda_{43}(j) /(j+1) \\
& =\left(\frac{2}{3}\right)\left\{0+\frac{40000}{2}-\frac{8000}{3}-\frac{4800}{4}+\frac{1600}{5}-\frac{128}{6}\right\}=\frac{98592}{9} .
\end{aligned}
$$

In a similar manner, we obtain

$$
I_{L}^{3.1}=\int_{l_{31}} \frac{x^{3} y}{3}(4 y-20) d y=\frac{-54820}{9} .
$$

Thus, from (123), (128) and (129), we obtain

$$
\iint_{\tau_{431}^{x y}} x^{2} y(4 y-20) d x d y=\frac{43772}{9} \text {. }
$$

Let us now consider

$$
\begin{aligned}
\iint_{\tau_{123}^{x}} x^{2} y(8 x-8 y) d x d y & =\iint_{\tau_{123}^{x y}} \frac{\partial}{\partial x}\left\{y\left(8 \frac{x^{4}}{4}-8 \frac{x^{3}}{3}\right)\right\} d x d y \\
& =\left(\int_{l_{12}}+\int_{l_{23}}+\int_{l_{31}}\right)\left(\frac{8}{4} x^{4} y-\frac{8}{3} x^{3} y^{2}\right) d y \\
& =\sum_{(p, q) \in\{(1,2),(2,3) \cdot(3,1)\}} I_{L}^{p q} .
\end{aligned}
$$

Clearly

$$
I_{L}^{p q}=\int_{l_{p q}}\left(\frac{8}{4} x^{4} y-\frac{8}{3} x^{3} y^{2}\right) d y .
$$

Using Lemma 3, for $\gamma+1=1, \alpha=2, \beta=1, l=8, m=-8$ gives

$$
I_{L}^{p q}=y_{p q}\left\{A_{p}(0,1) F^{p q}(3,1)+B_{p q}(0,1) H^{p q}(3,1)\right\},
$$


where

$$
\begin{gathered}
A_{p}(0,1)=\left(-\frac{8}{3} y_{p}+\frac{8}{4} x_{p}\right), \\
B_{p q}(0,1)=\left(-\frac{8}{3} y_{p q}+\frac{8}{4} x_{p q}\right) .
\end{gathered}
$$

Also using Lemma 5,

$$
\begin{aligned}
& F^{p q}(3,1)=\sum_{k=0}^{4} \frac{1}{(k+1)} \sum_{\lambda=0}^{k} a_{3, \lambda}^{p q} b_{1, k-\lambda}^{p q} \quad(0 \leq k-\lambda \leq 1,0 \leq \lambda \leq 3) \\
& =a_{3,0}^{p q} b_{1,0}^{p q}+\frac{1}{2}\left\{a_{3,0}^{p q} b_{1,1}^{p q}+a_{3,1}^{p q} b_{1,0}^{p q}\right\}+\frac{1}{3}\left\{a_{3,1}^{p q} b_{1,1}^{p q}+a_{3,2}^{p q} b_{1,0}^{p q}\right\} \\
& +\frac{1}{4}\left\{a_{3.2}^{p q} b_{1,1}^{p q}+a_{3.3}^{p q} b_{1.0}^{p q}\right\}+\frac{1}{5} a_{3.3}^{p q} b_{1.1}^{p q}, \\
& H^{p q}(3,1)=\sum_{k=0}^{4} \frac{1}{(k+2)} \sum_{\lambda=0}^{k} a_{3, \lambda}^{p q} b_{1, k-\lambda}^{p q} \quad(0 \leq k-\lambda \leq 1,0 \leq \lambda \leq 3) \\
& =\frac{1}{2} a_{3,0}^{p q} b_{1.0}^{p q}+\frac{1}{3}\left\{a_{3,0}^{p q} b_{1,1}^{p q}+a_{3.1}^{p q} b_{1.0}^{p q}\right\}+\frac{1}{4}\left\{a_{3,1}^{p q} b_{1,1}^{p q}+a_{3,2}^{p q} b_{1,0}^{p q}\right\} \\
& +\frac{1}{5}\left\{a_{3,2}^{p q} b_{1,1}^{p q}+a_{3,3}^{p q} b_{1,0}^{p q}\right\}+\frac{1}{6} a_{3,3}^{p q} b_{1,1}^{p q} \\
& a_{3.0}^{p q}=x_{p}^{3}, \quad a_{31}^{p q}=3 x_{p}^{2} x_{p q}, \quad a_{32}^{p q}=3 x_{p} x_{p q}^{2}, \quad a_{33}^{p q}=x_{p q}^{3}, \\
& b_{1,0}^{p q}=y_{p}, \quad b_{1,1}^{p q}=y_{p q} \text {. }
\end{aligned}
$$

We have from (111), (134), (135), (136) and (137) that

$$
\begin{aligned}
x_{1}=5, y_{1}=5, & x_{2}=10, y_{2}=10, \quad x_{3}=8, y_{3}=7, \\
x_{12}=x_{2}-x_{1}=5, & y_{12}=y_{2}-y_{1}=5, \\
x_{23}=x_{3}-x_{2}=-2, & y_{23}=y_{3}-y_{2}=-3, \\
x_{31}=x_{1}-x_{3}=-3, & y_{31}=-3, \\
A_{1}(0,1)=-\frac{10}{3}, & B_{12}(0,1)=-\frac{10}{3}, \\
A_{2}(0,1)=-\frac{20}{3}, & B_{23}(0,1)=4, \\
A_{3}(0,1)=-\frac{8}{3}, & B_{31}(0,1)=-\frac{2}{3}
\end{aligned}
$$




$$
\begin{aligned}
& F^{12}(3,1)=5^{3} \times 31, H^{12}(3,1)=5^{3} \times \frac{129}{6} \\
& F^{23}(3,1)=\frac{127896}{20}, H^{23}(3,1)=\frac{31992}{12} \\
& F^{31}(3,1)=\frac{146624}{15} \times \frac{3}{16}, \quad H^{31}(3,1)=\frac{41044}{45} \times \frac{3}{4} \\
& \int_{l_{12}\left(\frac{8}{4} x^{4} y-\frac{8}{3} x^{3} y^{2}\right) d y}=\frac{-50}{3}\left\{F^{12}(3,1)+H^{12}(3,1)\right\} \\
&\left.\int_{l_{23}\left(\frac{8}{4} x^{4} y-\frac{8}{3} x^{3} y^{2}\right) d y}=\frac{-109375}{3}, \frac{-20}{3} F^{23}(3,1)+4 H^{23}(3,1)\right\} \\
& \int_{l_{31}\left(\frac{8}{4} x^{4} y-\frac{8}{3} x^{3} y^{2}\right) d y}=(-2)\left\{\frac{-8}{3} F^{31}(3,1)-\frac{2}{3} H^{31}(3,1)\right\} \\
&=\frac{94268}{9} .
\end{aligned}
$$

Thus, from (139)-(141), we obtain from (131) that

$$
\iint_{\tau_{123}^{x y}} x^{2} y(8 x-8 y) d x d y=-\frac{26971}{9} .
$$

Finally from (113), (122), (120) and (142) we obtain

$$
\begin{aligned}
\iiint_{V} x^{2} y d x d y d z= & \iint_{\tau_{2: 4}^{x y}} x^{2} y\{-4(x-10)\} d x d y+\iint_{\tau_{43.1}^{*}} x^{2} y\{4 y-20\} d x d y \\
& -\iint_{\tau_{12.3}^{*}} x^{2} y(8 x-8 y) d x d y \\
= & \frac{23584}{3}+\frac{43772}{9}+\frac{26971}{9}=\frac{47165}{3}
\end{aligned}
$$

4.2. Algorithm 2 We shall now illustrate the use of Lemma 6 to compute integrals of the type

$$
\iint_{\tau_{i j j}^{x y}} x^{\alpha} y^{\beta}(l x+m y+h)^{\gamma+1} d x d y .
$$

Comparing with Lemma 6 for the application example (113), we have $\alpha=2, \beta=1$, $\gamma=0$ and here it will be sufficient to demonstrate the computational methodology with reference to one of the integrals, say

$$
\iint_{\tau_{2,34}^{r y}} x^{2} y\{-4(x-10)\} d x d y=I I_{\tau_{234}^{*}}^{2.1 .0}(-4,0,40) \text {. }
$$


We have from (61) and (62) that

$$
\begin{aligned}
I I_{\tau_{i j k}^{x}}^{2,1.0}(l, m, h) & =2 \Delta_{i j k} \sum_{n=0}^{4} \frac{S_{n}}{n+2} \\
& =2 \Delta_{i j k}\left\{\frac{S_{0}}{2}+\frac{S_{1}}{6}+\frac{S_{2}}{24}+\frac{S_{3}}{120}+\frac{S_{4}}{720}\right\},
\end{aligned}
$$

where

$$
\begin{aligned}
\frac{S_{0}}{2} & =\frac{\lambda(0,0)}{2}, \quad \frac{S_{1}}{6}=\frac{1}{6}\{\lambda(1,0)+\lambda(0,1)\}, \\
\frac{S_{2}}{24} & =\frac{1}{12}\{\lambda(2,0)+\lambda(0,2)\}+\frac{\lambda(1,1)}{24}, \\
\frac{S_{3}}{120} & =\frac{1}{60}\{\lambda(1,2)+\lambda(2,1)\}+\frac{1}{20}\{\lambda(3,0)+\lambda(0,3)\}, \\
\frac{S_{4}}{720} & =\frac{1}{180} \lambda(2,2)+\frac{1}{30}\{\lambda(4,0)+\lambda(0,4)\}+\frac{1}{120}\{\lambda(1,3)+\lambda(3,1)\}
\end{aligned}
$$

and

$$
\begin{gathered}
\lambda(p, q)=\sum_{p_{1}=0}^{p} \sum_{p_{2}=0}^{p-p_{1}} \sum_{q_{1}=0}^{q} \sum_{q_{2}=0}^{q-q_{1}} F\left(p_{1}, q_{1}\right) G\left(p_{2}, q_{2}\right) H\left(p_{3}, q_{3}\right) \\
\left(0 \leq p_{1}+q_{1} \leq 2=\alpha, \quad 0 \leq p_{2}+q_{2} \leq 1=\beta, \quad 0 \leq p_{3}+q_{3} \leq 1=\gamma+1\right)
\end{gathered}
$$

with

$$
\begin{array}{rlrl}
F(0,0) & =x_{i}^{2}, & G(0,0)=y_{i}, & H(0,0)=z_{i}, \\
F(1,0) & =2 x_{i} a_{x}, & G(1,0)=a_{y}, & H(1,0)=a_{z}, \\
F(0,1) & =2 x_{i} b_{x}, & G(0,1)=b_{y}, & H(0,1)=b_{z}, \\
F(2,0) & =a_{x}^{2}, & \\
F(0,2) & =b_{x}^{2}, & \\
F(1,1) & =2 a_{x} b_{x}, & \\
z_{i}=l x_{i}+m y_{i}+h, & z_{j}=l x_{j}+m y_{j}+h, & z_{k}=l x_{k}+m y_{k}+h, \\
a_{x}=x_{j}-x_{i}, & a_{y}=y_{j}-y_{i}, & a_{z}=z_{j}-z_{i}, \\
b_{x}=x_{k}-x_{i}, & b_{y}=y_{k}-y_{i}, & b_{z}=z_{k}-z_{i} .
\end{array}
$$

Letting $i=2, j=3, k=4$, we can evaluate the integral $I U_{\tau_{234}^{*}}^{2.1 .0}(-4,0,4,0)$ from (145)-(147). 
From (111), (147) and (148) we obtain

$$
\begin{gathered}
x_{2}=10, \quad x_{3}=8, x_{4}=10, \quad y_{2}=10, \quad y_{3}=7, \quad y_{4}=5, \quad z_{2}=0, z_{3}=8, z_{4}=0, \\
a_{x}=-2, \quad a_{y}=-3, \quad a_{z}=8, \\
b_{x}=0, \quad b_{y}=-5, \quad b_{z}=0 .
\end{gathered}
$$

Substituting the numerical values obtained from (149) into (147), we obtain

$$
\begin{array}{lll}
F(0,0)=100, & G(0,0)=10, & H(0,0)=0, \\
F(1,0)=-40, & G(1,0)=-3, & H(1,0)=8, \\
F(0,1)=0, & G(0,1)=-5, & H(0,1)=0, \\
F(2,0)=4, & & \\
F(0,2)=0, & \\
F(1,1)=0 . &
\end{array}
$$

Also from (63) we obtain

$$
2 \Delta_{234}=10
$$

$$
\begin{aligned}
& \lambda(0,0)=0, \quad \lambda(1,0)=8000, \quad \lambda(0,1)=0, \quad \lambda(1,1)=-4000, \\
& \lambda(2,0)=-5600, \quad \lambda(0,2)=0, \quad \lambda(2,1)=1600, \quad \lambda(1,2)=0, \\
& \lambda(3,0)=1280, \quad \lambda(0,3)=0, \quad \lambda(0,4)=0, \quad \lambda(4,0)=-96 \text {, } \\
& \lambda(2,2)=0, \quad \lambda(1,3)=0, \quad \lambda(3,1)=-160, \\
& \frac{S_{0}}{2}=\frac{1}{2} \times 0, \quad \frac{S_{1}}{6}=\frac{1}{6}\{8000+0\}, \quad \frac{S_{2}}{24}=\frac{1}{24}\{-4000\}+\frac{1}{12}\{0-5600\} \\
& \frac{S_{3}}{120}=\frac{1}{60}\{0+1600\}+\frac{1}{20}\{1280+0\}, \\
& \frac{S_{4}}{720}=\frac{1}{180} \times 0+\frac{1}{30}\{-96+0\}+\frac{1}{120}\{0-160\} \text {. }
\end{aligned}
$$

Finally, substituting from (151) and (152) into (145), we obtain

$$
\begin{aligned}
I I_{\tau_{234}}^{2,1,0}(-4,0,40)=(10) & {\left[\frac{0}{2}+\frac{8000}{6}+\frac{(-4000)}{24}+\frac{(-5600)}{12}+\frac{(1600)}{60}\right.} \\
& \left.+\frac{1280}{20}+\frac{0}{180}+\frac{(-96)}{30}+\frac{(-160)}{120}\right]
\end{aligned}
$$




$$
=\frac{23584}{3} \text {. }
$$

In a similar manner, on using (145)-(148), (111), (113) and (114) we obtain,

$$
\begin{gathered}
I I_{\tau_{1,3}^{* 113}}^{2,1.0}(8,-8,0)=\frac{-26971}{9}, \\
I I_{\tau_{4,31}^{2.1 .0}}^{2,1.0}(0,4,-20)=\frac{43772}{9} .
\end{gathered}
$$

From (153), (154), (155) and (113) we again find that

$$
\iiint_{V} x^{2} y d x d y d z=\frac{23584}{3}+\frac{26984}{9}+\frac{43772}{9}=\frac{47165}{3} .
$$

The results obtained in (143) and (156) are also in full agreement with that of Bernardini [1].

\section{Conclusions}

The theorem we have presented in this paper is interesting for various reasons as it has provided us with a powerful method to compute volume integration of trivariate polynomials as a sum of linear combinations of surface integrals of bivariate polynomial expressions in $\mathbb{R}^{2}$. In particular, the theorem proves that volume integration in $\mathbb{R}^{3}$ of the spatial trivariate polynomial $x^{\alpha} y^{\beta} z^{\gamma}(\alpha, \beta, \gamma$ are nonnegative integers) is related to the surface integration in $\mathbb{R}^{3}$ of the spatial expression $x^{\alpha} y^{\beta} z^{\gamma+1} /(\gamma+1)$ via triangulation of the linear polyhedral boundary. Further, the theorem establishes a relation between surface integration in $\mathbb{R}^{3}$ of the spatial expression $x^{\alpha} y^{\beta} z^{\gamma+1} /(\gamma+1)$ and a double integral over the $x y$-plane of the spatial expression $x^{\alpha} y^{\beta}(l x+m y+h)^{\gamma+1} /(\gamma+1)$.

We have also proposed a few lemmas (Lemmas 1 to 6 ) in which new techniques to integrate the spatial expression $x^{\alpha} y^{\beta}(l x+m y+h)^{\gamma+1}$ over the simple polygon or over the triangulation of the simple polygon in the $x y$-plane are considered. The proof of these lemmas has clearly demonstrated the efficient use of Taylor series expansions, Leibnitz's theorem on differentiation, Gauss's divergence theorem (Green's theorem) in two dimensions and the binomial theorem. Our formulas are more compact than those of previous researchers and require less computer arithmetic as is evident by comparing the summations required in earlier studies and the present one. These derivations are then followed by a numerical example for which we have explained the detailed computational scheme by use of two algorithms. The first algorithm computes a surface integral in $\mathbb{R}^{2}$ over an arbitrary triangle as a sum of three line integrals in a one-parameter space, while the second algorithm computes the same 
integral in $\mathbb{R}^{2}$ as a double integral over the unit triangle in two-parameter space. We have also developed a pseudocode for volume integration given in the appendix of the paper, which may help in implementing the method.

\section{Acknowledgements}

Professor H. T. Rathod is grateful to Professor J. F. Besseling who gave him the opportunity to pursue the research work during a one year postdoctoral Research Fellowship in 1990 at the Department of Mechanical Engineering, Technische Universiteit Delft, The Netherlands, as the research ideas on the above topic occurred to him during this period.

\section{Appendix: Pseudocode}

We feel that the development of a Pseudocode is quite simple if we follow the proof of the theorem on volume integration given in Section 3 of the paper.

Here is the simple outline of the Pseudocode.

(1) Find the equation of the plane spanned by each triangular surface $T_{f}$ of a polyhedron.

Let the number of such $T_{f}$ be denoted by $F$.

For $f=1$ to $F$

Compute the equation of the plane spanned by three vertices $\left(x_{i}^{f}, y_{i}^{f}, z_{i}^{f}\right)$, $\left(x_{j}^{f}, y_{j}^{f}, z_{j}^{f}\right)$ and $\left(x_{k}^{f}, y_{k}^{f}, z_{k}^{f}\right)$ for triangular surface $T_{f}$.

Determine the outward unit normal $\mathbf{n}_{f}$ and $K_{f}=\mathbf{k} \cdot \mathbf{n}_{f}$ [as a vector product $(0,0,1) \cdot\left(n_{1}, n_{2}, n_{3}\right)$ with $n_{3}=0$ or 1 or -1 .] Next $f$.

(2) Let $I V$ note the volume integral and $I S\left(F, K_{f}\right)$ the surface integral on triangular face $f$ with outward unit normal $\mathbf{n}_{f}$.

Compute $I V=\sum_{i=1}^{F} I S\left(f, K_{f}\right)$

where $I S\left(f, K_{f}\right)$ is computed via a function subprogram $I S\left(f, K_{f}\right)$.

(3) Function $I S\left(f, K_{f}\right)$.

Compute $I S\left(f, K_{f}\right)=K_{f} \iint_{\tau_{i j k}^{* x}} x^{\alpha} y^{\beta}(l x+m y+h)^{\gamma+1} d x d y$ by using Lemmas 1 to 5 or Lemma 6.

Return. 


\section{References}

[1] F. Bernardini, "Integration of polynomials over $n$-dimensional polyhedra", Comput. Aided Des. 23 (1) (1991) 51-58.

[2] C. Cattani and A. Paoluzzi, "Boundary integration over linear polyhedra", Comput. Aided Des. 22 (1990) 130-135.

[3] C. Cattani and A. Paoluzzi, "Symbolic analysis of linear polyhedra", Engineering with Computers 6 (1990) 17-29.

[4] Y. T. Lee and A. A. G. Requicha, "Algorithms for computing the volume and other integral properties of solids I : known methods and open issues", Comm. ACM 25 (9) (1982) 635-641.

[5] Y. T. Lee and A. A. G. Requicha, "Algorithms for computing the volume and other integral properties of solids II : a family of algorithms based on representation conversion and on cellular approximation", Comm. ACM 25 (9) (1982) 642-650.

[6] S. Lien and J. T. Kajiya, "A symbolic method for calculating the integral properties of arbitrary nonconvex polyhedra”, IEEE Comput. Graph. Applic. 4 (9) (1984) 35-41.

[7] J. R. O'Leary, "Evaluation of mass properties by finite elements", Guidance and Control 3 (2) (1980) 188-190.

[8] H. G. Timmer and J. M. Stern, "Computation of global geometric properties of solids", Comput. Aided Des. 12 (6) (1980) 301-304.

[9] H. B. Wilson, Jr and D. S. Farrior, "Computation of geometrical and inertial properties for general areas and volumes of revolution", Comput. Aided Des. 8 (4) (1976) 257-263. 